\title{
Outre : Art rupestre historique et sources écrites anciennes
}

\section{Malika Hachid}

\section{(2) OpenEdition}

1 Journals

Édition électronique

URL : https://journals.openedition.org/encyclopedieberbere/2878

DOI : 10.4000/encyclopedieberbere. 2878

ISSN : 2262-7197

Éditeur

Peeters Publishers

\section{Édition imprimée}

Date de publication : 2 juin 2013

Pagination : 6004-6009

ISBN : 978-2-7584-0194-0

ISSN : 1015-7344

\section{Référence électronique}

Malika Hachid, «Outre : Art rupestre historique et sources écrites anciennes », Encyclopédie berbère [En ligne], 36 | 2013, document 059, mis en ligne le 12 mars 2021, consulté le 17 février 2022. URL : http:// journals.openedition.org/encyclopedieberbere/2878; DOI : https://doi.org/10.4000/ encyclopedieberbere.2878

Ce document a été généré automatiquement le 17 février 2022.

(c) Tous droits réservés 


\title{
Outre : Art rupestre historique et sources écrites anciennes
}

\author{
Malika Hachid
}

L'art rupestre historique atlasique et saharien figure des outres d'eau sous-ventrières portées par des chevaux et des dromadaires. Celles des sites Rosfat men-el-Taht et "Strabon" (Atlas saharien, Algérie), bien que découvertes anciennement (en 1936 par R. Vaufrey pour le premier, et en 1967 par feu J. Iliou, pour le second) ont plus tard été identifiées comme telles grâce à un témoignage de Strabon ( $1^{\mathrm{er}}$ siècle av. J.-C. / $1^{\mathrm{er}}$ siècle après) (Hachid 1992, II, fig. 276 et fig. 291 à 294) [fig. 1]. Développant ce sujet à travers le recoupement des sources écrites et rupestres, nous avons rebaptisé le second site du nom de cet auteur ancien (Hachid 2011). D'autres sites rupestres reproduisant ce mode de transport dans le Sahara mauritanien, le Tassili-n-Ajjer (sud algérien) et la Tadrart Akakus (sud du Fezzan, Libye) montrent qu'il était en usage à travers tout le Sahara (Hachid 2011). 


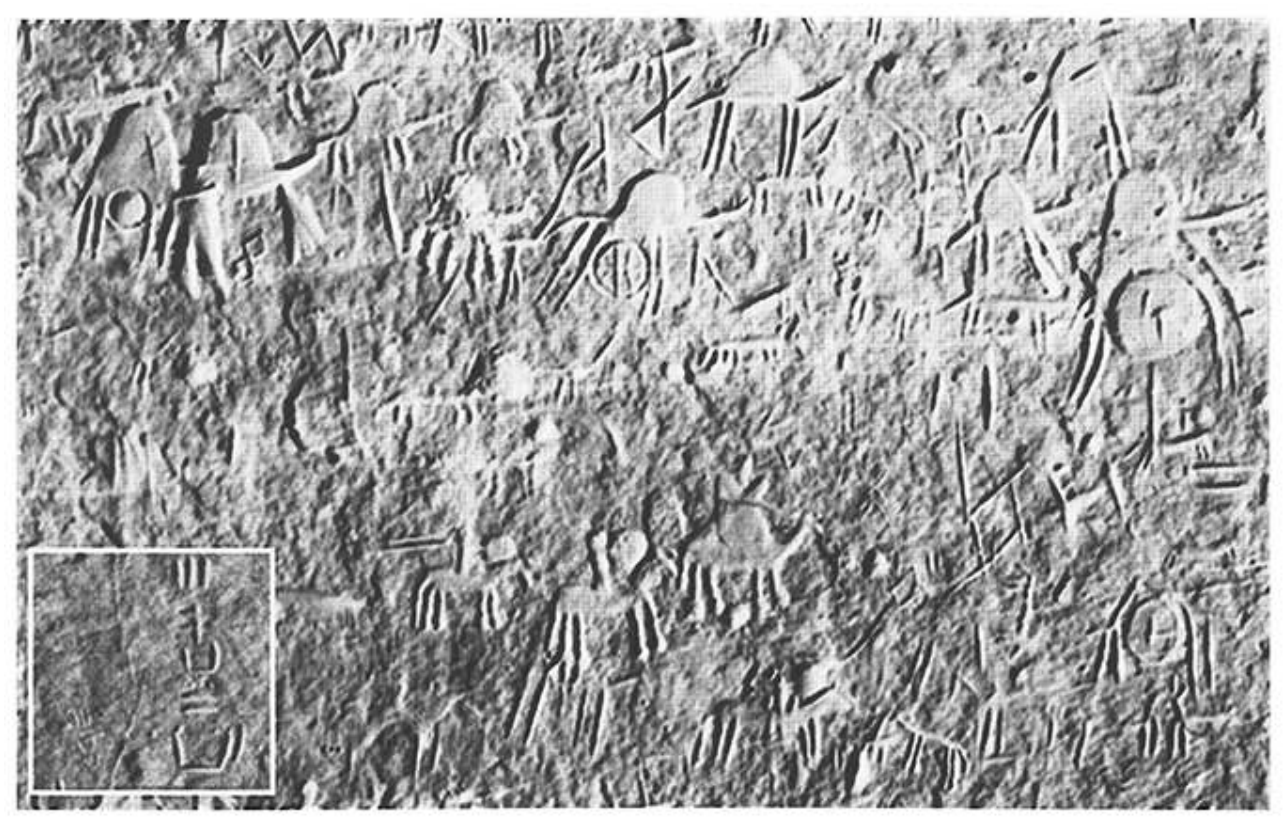

Fig 1 : Site "STRABON" (MONTS DES KSOUR, ATLAS SAHARIEN, ALgÉRIE) (1: 1,50 M)

Cliché M. Hachid.

\section{Les textes gréco-latins}

2 Les armées assyriennes puis perses qui envahirent l'Egypte et la Cyrénaïque (VIII ${ }^{e}$ et $\mathrm{VI}^{\mathrm{e}}$ siècle avant $\mathrm{J}$.-C.) utilisaient des dromadaires prêtés par les alliés arabes pour transporter eau, vivres et autre matériel (Hérodote, $V^{e}$ siècle avant J.-C.). En 332 avant J.-C., lors du pèlerinage qu'Alexandre le Grand fit à l'oasis de Siwa* (Désert Libyque), ce sont encore des dromadaires amenés d'Orient qui transportaient des outres d'eau sur le dos (Quinte-Curce, $1^{\mathrm{er}}$ siècle après J.-C. ; L'Histoire d'Alexandre le Grand, IV, VII). Relatant la «Guerre de Jugurtha » qui se déroula entre 111 et 105 avant J.-C., Salluste $\left(1^{\text {er }}\right.$ siècle avant J.-C.) écrit que lorsque Métellus décida d'aller prendre Thala (sud-tunisien actuel), la capitale du chef berbère qui s'y était replié, il n'ignorait pas qu'il allait devoir traverser une importante zone désertique avant d'atteindre une rivière proche de cette cité : "Il fait donc enlever aux bêtes de somme leurs fardeaux, ne leur laisse que des sacs de blé pour dix jours, des outres et d'autres vases " (LXXV). Enfin, Strabon, au début de l'ère chrétienne, en nous apprenant que « Les Pharusiens se rencontrent avec les Maures à travers le désert, bien que rarement, en attachant des outres d'eau sous le ventre de leurs chevaux » évoque clairement un mode de transport autre que celui qui pouvait se faire à dos de bêtes, que celles-ci aient été le cheval, le dromadaire ou l'âne (Géographie, XVII, III, VII).

\section{Les témoignages arabes}

3 À la fin du VII siècle (666-667), le conquérant arabe 'Oqba Ibn Nafi* se lança à la conquête du Fezzan et du Kawar avec une troupe de 400 cavaliers et 400 chameaux, ces derniers portant chacun deux outres d'eau (Ibn Abd El-Hakam, IX ${ }^{\mathrm{e}}$ siècle). Au XII ${ }^{\mathrm{e}}$ siècle, Al-Idrisi fait état du même transport par les caravanes qui traversaient la majaba ou désert de Nisar, au sud de la piste qui s'étendait de Sijilmassa à Awdaghost/Tegdaoust 
vers le royaume de Ghana, une étape de 14 jours sans points d'eau (1968, p. 198). En 1590-1591, quittant le Sud marocain pour traverser le désert dans le but de soumettre l'empire de Gao, l'armée du sultan du Maroc comptait de nombreux chevaux qui étaient abreuvés grâce aux outres portées par les dromadaires. De nos jours encore, les Maures comme les Touaregs du Sud utilisent des outres arrimées au ventre des ânes ou des bœufs (Bernus et Durou 1996, p. 238).

\section{L'ancienneté des outres sous-ventrières dans l'art rupestre historique}

4 Le transport de ballots et de sacs à dos de boeufs porteurs, renforcé par des sangles passées sous le ventre et la queue de l'animal, est visible dans l'art rupestre des Protoberbères bovidiens (style d'Eheren et Wa-n-Amil). Le transport de l'eau dans des outres a dû se développer avec l'accroissement de l'aridité et on sait que la dernière phase aride post-néolithique débute vers le IVe millénaire BP. Ce procédé a dû apparaitre spontanément en divers endroits de la Libye des Anciens comme du Proche-Orient antique, là où des peuples nomades se déplaçaient dans des régions exposées à la sécheresse. L'outre sous-ventrière a d'abord été transportée par le cheval avant l'introduction du dromadaire, sans que l'on puisse dire à quel moment précis, sinon que le texte de Strabon semble être le plus ancien à mentionner le cheval.

Le dromadaire étant connu au Sahara central vers les III ${ }^{\mathrm{e}}$-IV ${ }^{\mathrm{e}}$ siècles (Elachid 2006), les plus anciennes représentations rupestres d'outres sous-ventrières sahariennes arrimées à cet animal remonteraient à cette même époque. Le site "Strabon" figure des inscriptions libyques qui pourraient se situer à une date comprise entre la fin de l'Antiquité byzantine et le début des premières conquêtes musulmanes, soit vers les $\mathrm{VI}^{\mathrm{e}}$-VII ${ }^{\mathrm{e}}$ siècles, une date un peu plus tardive que les $\mathrm{V}^{\mathrm{e}}-\mathrm{VI} \mathrm{e}^{\mathrm{e}}$ siècles qui auraient vu la disparition des alphabets libyques (Hachid 2011, p. 211 à 212). La caravane représentée, où les dromadaires sont plus nombreux que les cavaliers armés chargés de la protéger, pourrait figurer ce petit négoce transsaharien avec l'Afrique byzantine dont quelques historiens estiment qu'il pourrait remonter au $\mathrm{VI}^{e}$ siècle AD. Il n'est pas impossible qu'elle puisse aussi évoquer les débuts du grand commerce caravanier transsaharien de la période dite "médiévale » dont on sait grâce à El-Bekri $\left(\mathrm{XI}^{\mathrm{e}}\right)$ et surtout Abu Zakariya el-Warglani (XII $)$ qu'il se met en place dès le VIII ${ }^{e}$ siècle (El-Bekri 1965 ; Le Tourneau 1960, 1961). 


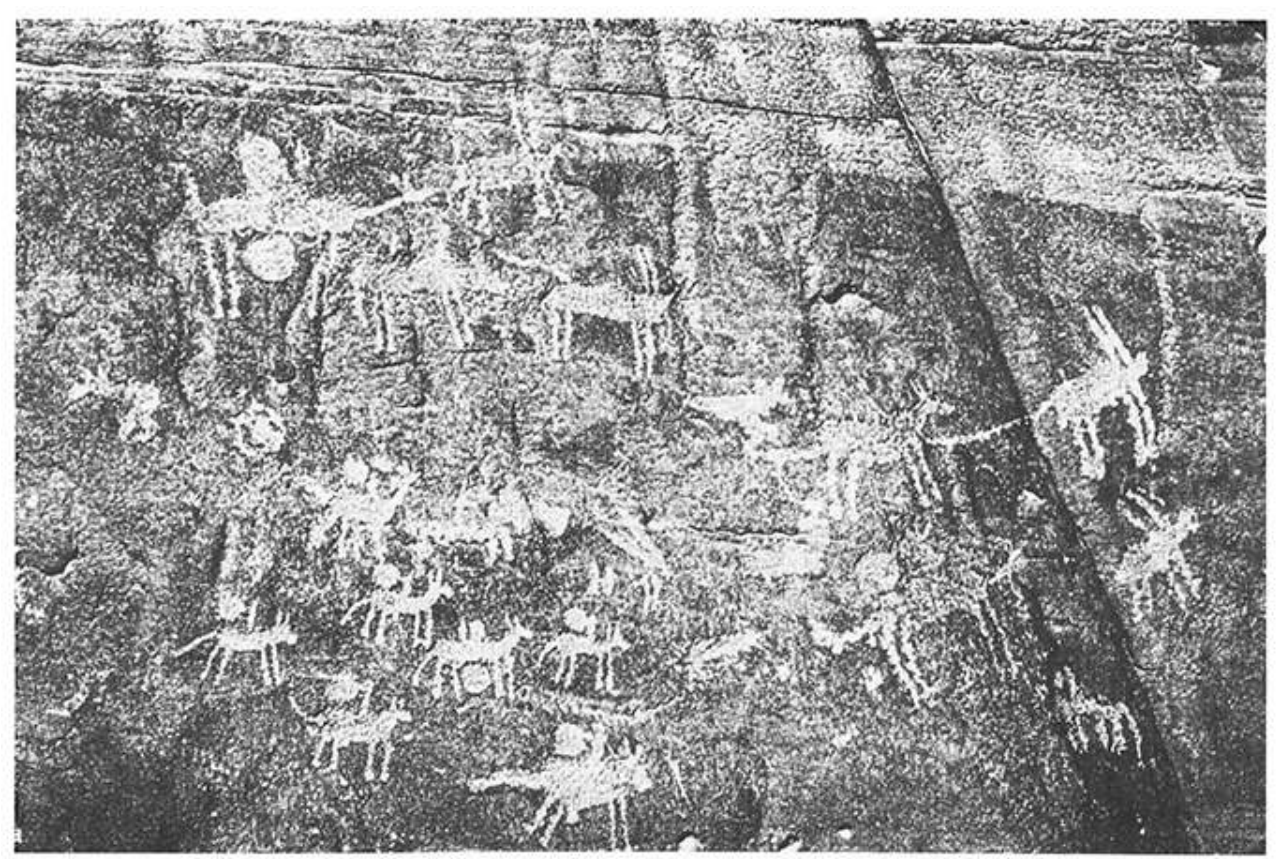

FIg 2 : GATA'NÂAM (HODH SEPTENTRIONAL, SUD-EST MAURITANIEN) (AMBLARD 1993, P. 50), 1993.

6 Dans la scène de chasse à l'antilope oryx de Gata' Nâam (Hachid 2011, p. 198) [fig. 2], la forme circulaire et les petites dimensions de la rondache des cavaliers ne fournit aucune indication d'ordre chronologique car ce type de bouclier qui remonte à l'Antiquité a inexplicablement perduré dans l'art rupestre historique jusqu'aux temps subactuels, alors que les Touaregs font usage de grands boucliers quadrangulaires (Hachid, à paraître). Cependant, Gata'Nâam illustre un témoignage qui revient souvent dans les sources arabes, celui du bouclier dit lemta, confectionné dans la peau, très solide, de l'oryx. Le site se trouve dans le désert mauritanien dont les pistes, comme celles du Sahara occidental, étaient sous le contrôle de grandes tribus sanhadja*; la plus anciennement révélée par les textes arabes, par Al-Fazari, au VIII ${ }^{\mathrm{e}}$ siècle, est celle des Anbiya* qui fabriquaient déjà ce type de bouclier (Ibn el-Faqih, 1886). Aussi, cette scène de chasse avec son équipement en outres sous-ventrières pourrait-elle remonter à la période « médiévale » sans que l'on puisse davantage en préciser la période exacte.

\section{Les outres sous-ventrières rupestres et leurs implications pour le peuplement du Sahara}

7 Une première implication est celle de la localisation géographique du territoire des Pharusiens. Chez Pline l'Ancien ( $1^{\text {er }}$ siècle avant J.C.) qui rapporte le témoignage perdu du périple de Polybe sur l'exploration de la côte atlantique en 146 avant J.-C., ils se tenaient au sud de l'oued Darat dans lequel les historiens ont reconnu l'oued Drâa actuel, au sud du Maroc (Histoire naturelle, V, I). Un peu plus tard, chez Strabon, ils sont également localisés à la latitude de l'oued Drâa, alors que le territoire des Maures s'arrêtait, au sud, à celle du Moyen Atlas (Géographie, XVII, III, VII ; Desanges 1989, 2001). Strabon ajoute que depuis leur territoires méridionaux, les Pharusiens faisaient des incursions pour attaquer les établissements carthaginois sur la côte atlantique, alors qu'à l'est, ils "poussaient» jusqu'à Cirta (actuelle Constantine), la capitale numide (Géographie, XVII, III, VII). La latitude de l'oued Drâa nous parait être trop 
septentrionale. Cette région était certes semi-désertique, mais, par divers témoignages gréco-latins, et notamment ceux de Pline l'Ancien (Histoire naturelle, V, I) et de Strabon qui cite Iphicrate (IVe siècle avant J.-C.), on sait qu'elle était aussi pourvue de points d'eau et, surtout, elle abritait l'oued du même nom où vivaient des crocodiles, tandis que ses rives étaient habités par divers peuples (Géographie, XVII, III, VII). Par ailleurs, on aura remarqué que lorsqu'il évoque les outres sous-ventrières, Strabon les met en relation avec les Pharusiens seulement et pas avec les Maures. Si ceux-ci devaient partir d'un territoire situé à la latitude de l'Oued Drâa, qui ne manquait pas d'eau, pour gagner un lieu de rencontre se situant plus au nord, ces cavaliers avaient-ils besoin de s'équiper d'outres d'eau? Il est fort possible que si les Pharusiens prévoyaient de telles réserves, c'est parce qu'ils devaient venir d'une région plus méridionale et qu'ils devaient traverser des zones arides avant d'atteindre le lieu où les Maures les attendaient. Leur territoire semblerait plutôt se situer au sud de l'oued Drâa et, au moins, au nord du Sahara occidental et/ou du Désert mauritanien.

a seconde implication de ce mode de transport est, bien sûr, celle d'une certaine mobilité des Sahariens avant même l'acquisition du dromadaire. Grâce à une bonne connaissance des pistes et des points d'eau, des déplacements de proche en proche et des échanges intra-sahariens étaient possibles d'est en ouest et du nord au sud du Sahara. Quant aux longues traversées de ce désert, même si elles furent rares, les Sahariens, du moins leurs élites, en ont néanmoins accomplies comme en témoignent les sources écrites, l'art rupestre et les documents archéologiques qui font état de leurs contacts avec les deux rives du Sahara, Méditerranée, au nord, Sahel, au sud (Hachid 2007 ; 2011). Les populations du Sahara étaient beaucoup moins isolées qu'on l'a souvent supposé.

Dans l'état actuel des connaissances, l'usage de l'outre est donc attesté par les textes gréco-latins et arabes et les représentations rupestres d'âge historique, depuis la région de l'Atlas saharien jusqu'à des régions sahariennes telles que le Désert mauritanien, le Tassili-n-Ajjer et la Tadrart Akakus. Il remonte à l'Antiquité, où les textes lui font jouer un rôle décisif au cours des batailles et des guerres, traverse l'époque " médiévale » et s'est conservé jusqu'à nos jours. Si son insertion est pérennisée, depuis une date indéterminée, dans les usages quotidiens et les déplacements des sociétés montagnardes du bassin méditerranéen (relief accidenté à franchir) en relation avec le transport par mulets et ânes, dans les régions méridionales, il sert notamment de repère climatique (désertification), géographique (région aride à subaride) et, enfin, économique, en relation avec l'activité domestique, cynégétique et caravanière.

\section{BIBLIOGRAPHIE}

AL-IDRISI, 1968 - Description de l'Afrique et de l'Espagne, éd. R. Dozy et M.J. De Goeje, Leyde, 393 p. + texte arabe ( $1^{\text {ère }}$ impression en 1864-1866). 
AMBLARD S., 1993 - « Les gravures rupestres du Hodh septentrional (Mauritanie sud-orientale) », L'Arte et l'Ambiente del Sahara preistorico : dati e interpretaziono, Milano 24-27 ottobre 1990, p. 41 à 51.

EL-BEKRI, 1965 - Kitâb el-Massâlik ou el-Mamâlik, traduit par baron MacGuckin de Slane, réédition de l'édition de 1913 revue et corrigée, Paris, Maisonneuve et Larose.

BERNUS E. et DURROU J.-M., 1996 - Touaregs. Un peuple du désert, Paris, Robert Laffont, 331 p.

DESANGES J., 1989 - « Atlas », Encyclopédie berbère, VII, p. 1013 à 1015.

DESANGES J., 2001 - « Le témoignage de Strabon (XVII, 3, 2-8) sur la Maurousie et l'Ethiopie occidentale ", Actes des 1ères Journées d'Archéologie et du Patrimoine (juillet 1998), Rabat, p. 19 à 24.

HACHID M., 1992 - El-Hadjra el-Mektouba. Les Pierres écrites de l'Atlas saharien, I, 176 p., II, 343

illustrations, Alger, ENAG.

HACHID M., 2006 - «Du nouveau sur le monument d'Abalessa (Ahaggar, Algérie). De la date de l'introduction du dromadaire au Sahara central, du personnage d'Abalessa et des inscriptions rupestres dites "libyco-berbères" ", Sahara, 17, p. 95 à 120.

HACHID M., 2011 - «Strabon, El-Idrissi, la Guerba et un Libyque plus tardif que les Ve/ $\mathrm{VI}^{\mathrm{e}}$ siècles? ", Actes du Premier Colloque de Préhistoire maghrébine, Tamanghasset, 5-7 novembre 2007, Travaux du CNRPAH, n.s., 11, II, p. 191-225.

HACHID M., (à paraître) - « L'art rupestre historique pourrait-il représenter les descendants des Sanhadja et Huwwara, peuples chers à Marceau Gast? ", Hommage à Marceau Gast, (S. Chaker \& H. Claudot-Hawad éds.), Louvain, Peeters..

IBN 'ABD AL-HAKAM, 1947 - Conquête de l'Afrique du Nord et de l'Espagne (Futuh'Ifriqiya wa'l-Andalous), texte arabe et traduction française par Albert Gateau, $2^{\mathrm{e}}$ édition, Alger, Carbonel.

IBN EL-FAKIH, 1886 - Mukhtasar Kitâb el-Buldân, éd. M.J. de Goeje, Bibliotheca geographorum arabicorum, t. V, Paris.

LE TOURNEAU R., 1960 - « Abu Zakariyyâ al-Wargalanî "La chronique" », Revue Africaine, CIV, p. 99 à 176.

LE TOURNEAU R., 1961 - « Abu Zakariyyâ al-Wargalanî "La chronique” ", Revue Africaine, CV, p. 117 à 176.

INDEX

Mots-clés : Antiquité, Protohistoire, Sahara 